\title{
Pardeamiento enzimático: caracterización fenotípica, bioquímica y molecular en variedades de papa nativas de la Argentina
}

\author{
P.A. Suárez ${ }^{1}$, A.B. Andreu ${ }^{2}$, S.L. Colman ${ }^{3}$, A. M. Clausen ${ }^{4}$, S.E. Feingold ${ }^{5}$ \\ RESUMEN
}

El pardeamiento enzimático (PE) esta relacionado con la actividad de la enzima polifenol oxidasa (PPO) que cataliza la oxidación a diferentes compuestos fenólicos, con la consecuente transformación a pigmentos oscuros no deseables para la calidad industrial. Este trabajo contribuye a la caracterización del PE en papas nativas (Solanum tuberosum ssp. andigenum) a través de la determinación de la variabilidad alélica de los genes de PPO, la actividad enzimática relacionada y la resultante calidad industrial por medio de parámetros cualitativos. En los 20 genotipos estudiados se encontraron diferencias significativas en la actividad de PPO y el índice oxidativo (IO), lo que permitió distinguir individuos con mejor comportamiento frente al PE. Asimismo, se observó variabilidad genética en las regiones amplificadas de los genes de PPO identificándose 9 posibles variantes alélicas. Hasta el momento no se pudo establecer asociación entre bandas y la actividad de PPO o IO, posiblemente debido a factores ambientales que pudieron haber incidido durante el desarrollo del cultivo y/o al número reducido de genotipos estudiados a la fecha.

Palabras clave: pardeamiento enzimático, polifenol oxidasa, papas nativas, índice oxidativo

\section{Enzimatic browning: phenotypic, biochemical and molecular characterization of native potatoes from Argentina}

\begin{abstract}
Enzymatic browning (EB) is related to polyphenol oxidase (PPO) activity, which catalyzes the oxidation of various phenolic compounds that renders dark pigments which are undesirable for industrial quality of potato. This study contributes to the characterization of EB in native potatoes (Solanum tuberosum ssp. andigenum) through the analysis of allelic variability in PPO genes, the determination of the enzymatic activity and the resulting darkening of tubers through qualitative parameters. Variability was found among 20 selected genotypes in activity of PPO and oxidative index (OI), which allowed distinguishing individuals with better EB performance. Also, genetic variability was observed in the amplified regions of PPO genes, which shown 9 possible allelic variants. However, no clear association was found between

\footnotetext{
${ }^{1}$ Licenciada en Producción Vegetal. Técnico Profesional Adjunto Consejo Nacional de Investigaciones Científicas y Técnicas (CONICET), Instituto de Investigaciones Biológicas, Universidad Nacional de Mar del Plata (UNMdP), Funes 3250 (7600) Mar del Plata, Buenos Aires, Argentina.psuarez@ balcarce.inta.gov.ar

${ }^{2}$ Licenciada y Dra. en Ciencias Biológicas, Investigador Independiente CONICET, Instituto de Investigaciones Biológicas, UNMdP, Mar del Plata, Argentina. abandreu@mdp.edu.ar.

${ }^{3}$ Licenciada en Ciencias Biológicas, Becaria Doctoral CONICET, Estación Experimental Agropecuaria-Instituto Nacional de Tecnología Agropecuaria (EEA INTA) Balcarce, Ruta 226 Km 73.5. CC 276 (7620), Buenos Aires, Argentina.scolman@balcarce.inta.gov.ar

4 Ing. Agr. (MSc), Banco de Germoplasma Papa y Forrajeras, EEA INTA, Balcarce, Argentina. aclausen@balcarce.inta.gov.ar

${ }_{5}^{5}$ Ing. Agr. y Dr. en Ciencias Biológicas. Responsable del Laboratorio Agrobiotecnología, EEA INTA, Balcarce, Argentina. sfeingold@ balcarce.inta.gov.ar
} 
putative alleles and PPO activity or OI . This lack of association can be related to the reduced number of genotypes assayed up to date and/or environmental factors that could have influenced tuber production.

Key words: enzimatic browning, polyphenol oxidase, native potatoes, oxidative index

\section{Introducción}

Las variedades nativas de papa (Solanum tuberosum ssp. andigenum) que se cultivan en el noroeste argentino (NOA), tienen un valor fundamental en la alimentación de los pobladores andinos, y representan un importante potencial genético para el desarrollo de nuevos productos por sus características de calidad culinaria y agronómicas (Clausen, 1989).

Se ha reportado que las variedades nativas presentan bajo pardeamiento con respecto a las variedades comerciales (Álvarez-Mayorca, 2001), sin embargo, esta característica no ha sido estudiada en profundidad en las variedades nativas argentinas a la luz de la variabilidad presentada por genes candidatos. El color café que se forma al cortar $\mathrm{y} / \mathrm{o}$ maltratar los tubérculos se conoce como pardeamiento enzimático, ya que las reacciones iniciales que intervienen en este fenómeno están catalizadas por enzimas oxidasas.

El pardeamiento enzimático (PE) esta relacionado principalmente con la actividad de polifenol oxidasas (PPO), la cuales catalizan la oxidación de compuestos fenólicos a quinonas, con la consecuente transformación a pigmentos oscuros no deseables para la calidad industrial (Friedman, 1997). Las PPO están codificadas por seis genes denominados POTP1, POTP2, POT32, POT33, POT41 y POT72 (Hunt et al., 1993; Thygesen et al., 1995) situados en el cromosoma 8 (Werij et al., 2007). De ellos, tres son específicos de tejidos no fotosintéticos (POT32, POT33 y POT72), y el POT32 colocaliza con un locus de carácter cuantitativo (QTL) para el PE (Werij et al., 2007).

El objetivo de este trabajo es caracterizar el comportamiento frente al pardeamiento enzimático de papas nativas a través de análisis colorimétricos, estudio de los componentes bioquímicos relacionados al pardeamiento y el establecimiento de la variabilidad alélica en los genes de polifenoloxidasas específicas de tubérculo.

\section{Materiales y métodos}

Se evaluaron 20 variedades locales de Solanum tuberosum ssp. andigenum (adg) provenientes de distintas localidades del NOA y cultivadas para este estudio en invernáculo de la Estación Experimental Agropecuaria del Instituto Nacional de Tecnología Agropecuaria (EEA INTA) Balcarce, Buenos Aires, Argentina. Este material integra la colección de papas nativas del Banco de Germoplasma de Papa y Forrajeras, EEA INTA Balcarce.

Se determinó la actividad de PPO en 1 g de pulpa y cáscara de mini tubérculos por espectrofotometría (Chen et al., 2000) con dos repeticiones independientes. El extracto se homogenizó sobre hielo con un buffer de extracción, proporción 1:1 (peso / volumen), que contenía $\mathrm{PO}_{4} \mathrm{Na} 100 \mathrm{mM}$ a $\mathrm{PH} 7.5$; metabisulfito de $\mathrm{Na} 2 \mathrm{mM}$; ClNa $0.5 \mathrm{mM}$ y $1 \%$ de polyvinilpirrolidona (PVP). Posteriormente se centrifugó y se colectó el sobrenadante para luego determinar la actividad a $420 \mathrm{~nm}$ en espectrofotómetro. La reacción fue iniciada por la adición del sustrato 4-methyl catechol $2 \mathrm{mM}$ de concentración final, por el término de 1 minuto. El resultado se expresó en unidades/minuto por $\mathrm{g}$ de peso fresco (U/min $\mathrm{g}$ PF), como el incremento de absorbancia a 420 nm que produce $1 \mathrm{~g}$ de peso fresco en 1 minuto. Para evaluar el índice oxidativo (IO) se utilizó una carta de color del Instituto de Pflazembau und Saatgutforschung der FAL BraunschweigVolkenrode (Pätzold, 1973) y se aplicó la fórmula: $\mathrm{IO}=$ (valor intensidad del color-valor del color) +16 . Los cortes de papa de $10 \mathrm{~mm}$ de diámetro se evaluaron a los 15, 30, 45 y 60 minutos con dos repeticiones independientes. Los valores obtenidos oscilan en un rango entre 0 (marrón oscuro) y 24 (blanco). Por lo tanto, valores elevados representan cortes de papas de colores claros o una reducción en el PE. 
Se determinó la variabilidad alélica mediante la amplificación por PCR con dos juegos de iniciadores específicos (P-PO32-1, P-PO32-2, PPO33-1 y P-PO33-2) para cada una de las secuencias de ADNc de POT32 y POT33 del Potato Gene Index (Computational Biology and Functional Genomics Laboratory, 2008). Se evaluó el polimorfismo de conformación de hebra simple (SSCP), mediante electroforesis de los fragmentos resultantes en geles de poliacrilamida parcialmente nativos $\left(\mathrm{MDE}^{\circledR}\right.$ Cambrex, 2003). El Índice de Diversidad se calculó según Milbourne et al. (1997).

Se determinó la asociación entre actividad PPO,
PE y la variación alélica encontrada mediante el test estadístico de Kruskal Wallis. Los resultados análisis de varianza y las medias por pruebas de Test LSD Fisher utilizando el programa Infostat v. 2008 con un $\alpha=0,05$.

\section{Resultados y discusión}

La actividad de PPO mostró diferencias significativas entre variedades (Tabla 1) oscilando entre 215 y $1010 \mathrm{U} / \mathrm{min}$ g PF; donde Collareja (LC 328, LC 441, CL 621), Criolla (LC 262) y Blanca (LC 451) presentaron la mayor actividad de PPO y Moradita (CCS 1307) la menor. Los resultados de IO hallados en el estudio oscilaron entre 13 y 23 (Tabla 1).

Tabla 1. Actividad de polifenol oxidasas (PPO) e índice oxidativo en las variedades de Solanum tuberosum ssp. andigenum del noroeste argentino $^{\mathrm{a}}$

\begin{tabular}{|c|c|c|c|c|c|}
\hline \multirow{2}{*}{$\begin{array}{l}\text { Variedades } \\
\text { Collareja }\end{array}$} & \multirow{2}{*}{$\frac{\text { Acceso }}{\text { LC } 328}$} & \multicolumn{2}{|c|}{ PPO $(\mathrm{U} / \mathrm{min} \text { g PF })^{\mathrm{b}}$} & \multicolumn{2}{|c|}{ Índice oxidativo } \\
\hline & & 1010 & $\mathrm{~A}$ & 15 & $\mathrm{BC}$ \\
\hline Criolla & LC 262 & 960 & $\mathrm{AB}$ & 14 & \\
\hline Collareja & CL 621 & 853 & $\mathrm{AB}$ & 19 & $\mathrm{EF}$ \\
\hline Collareja & LC 441 & 800 & $\mathrm{ABC}$ & 17 & $\mathrm{D}$ \\
\hline Blanca & LC 451 & 688 & $\mathrm{BC}$ & 19 & $\mathrm{EF}$ \\
\hline Rara Cauqueva & CQA3 & 575 & $\mathrm{BCD}$ & 15 & $\mathrm{BC}$ \\
\hline Collareja & CS 1432 & 570 & $\mathrm{BCD}$ & 22 & $\mathrm{G}$ \\
\hline Rosada & CCS 1321 & 565 & $\mathrm{BCD}$ & 17 & $\mathrm{D}$ \\
\hline Overa & CCS 1194 & 564 & $\mathrm{BCD}$ & 13 & \\
\hline Chaqueña & LC 341 & 560 & $\mathrm{BCD}$ & 15 & $\mathrm{BC}$ \\
\hline Collareja & LC 344 & 560 & $\mathrm{BCD}$ & 14 & \\
\hline Colorada & LC 509 & 532 & $\mathrm{E}$ & 15 & $\mathrm{BC}$ \\
\hline Moradita & LC 82 & 498 & $\mathrm{EF}$ & 20 & $\mathrm{~F}$ \\
\hline Tuni Morada & LC 342 & 497 & $\mathrm{EF}$ & 15 & $\mathrm{BC}$ \\
\hline Collareja & LC 515 & 469 & EFG & 23 & $\mathrm{G}$ \\
\hline Rosada & LC 198 & 378 & FG & 22 & $\mathrm{G}$ \\
\hline Collareja & LC 482 & 357 & FG & 15 & $\mathrm{BC}$ \\
\hline Colorada & CCS 1395 & 339 & FG & 21 & G \\
\hline Imilla Negra & LC 348 & 274 & G & 16 & $\mathrm{BC}$ \\
\hline Moradita & CCS 1307 & 215 & $\mathrm{H}$ & 19 & $\mathrm{EF}$ \\
\hline
\end{tabular}


El análisis de SSCP presentó variabilidad alélica para los genes estudiados (Figura 1 y Tabla 2). Se hallaron al menos 9 variantes alélicas para el gen POT 32, el cual mostró la mayor variabilidad, mientras que el gen POT 33 evidencia 7 variantes alélicas. Los genotipos con baja actividad de PPO $(<350 \mathrm{U} / \mathrm{min}$ g PF $)$ presentaron patrones diferentes a aquellos con una alta actividad $(<800 \mathrm{U} / \mathrm{min}$ g PF), pero también distintos entre sí (Figura 1). Asimismo, los distintos genotipos de Collareja tuvieron valores de actividad de PPO entre 357 y 1010
U/min g PF, a pesar de presentar el mismo patrón genético para las cuatro regiones amplificadas. No se pudo establecer asociación entre las bandas de SSCP, la actividad de PPO y el IO. Éstos resultados no son del todo inesperados dada las condiciones no óptimas del desarrollo del cultivo. Esto concuerda con lo descripto por Porcel et al. (2006) donde se atribuye parte de la oxidación de los flavonoides por otros procesos en las plantas, tales como el desarrollo fisiológico de la planta, el estrés biótico y abiótico.

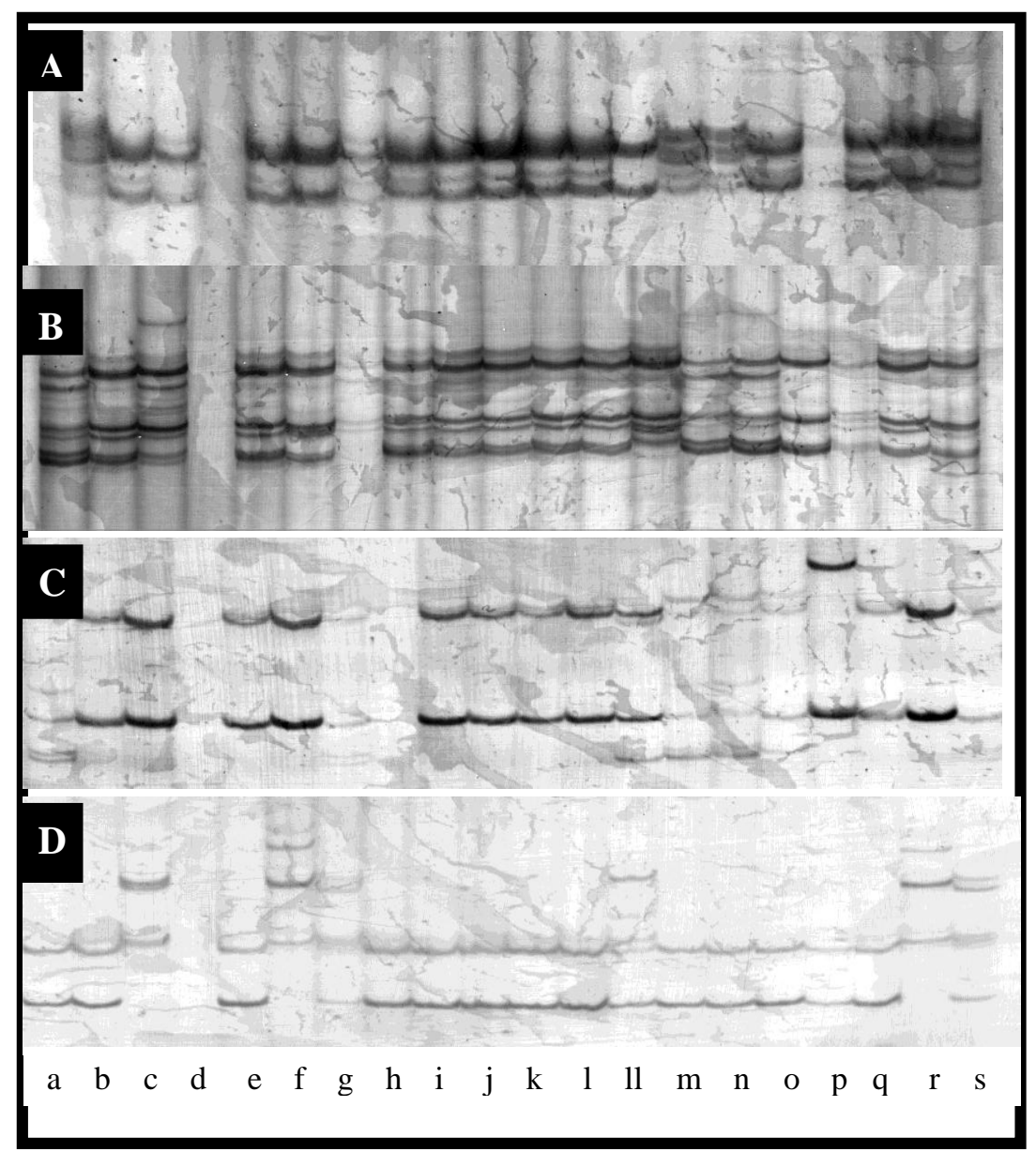

Figura 1. Orden de las variedades de Solanum tuberosum ssp. andigenum del noroeste argentino en geles SSCP para los genes POT 32 y POT 33. Iniciadores: A) P-PO32-1; B) P-PO32-2; C) P-PO33-1; D) P-PO33-2. Variedades: a) Rara Cauqueva CQA3; b) Collareja LC328; c) Criolla LC262; d) Chaqueña LC341; e) Collareja CS1432; f) Rosada LC198; g) Moradita LC82; h) Imilla Negra LC348; i) Collareja LC344; j) Collareja LC441; k) Collareja LC515; 1) Collareja CL621; 1l) Colorada LC509; m) Overa CCS1194; n) Tuni Morada LC342; o) Rosada CCS1321; p) Colorada CCS1395; q) Collareja LC482; r) Blanca LC451; y s) Moradita CCS1307, respectivamente, para cada iniciador. 
Tabla 2. Caracterización genotípica de las variedades de Solanum tuberosum ssp. andigenum del noroeste argentino mediante polimorfismo de conformación de hebra simple

\begin{tabular}{lcccccc}
\hline \multirow{2}{*}{ Características } & \multicolumn{2}{c}{ Gen POT 32 } & & \multicolumn{2}{c}{ Gen POT 33 } \\
\cline { 2 - 3 } \cline { 6 - 7 } & $\begin{array}{l}\text { Iniciador } \\
\text { P-PO32-1 }\end{array}$ & $\begin{array}{c}\text { Iniciador } \\
\text { P-PO32-2 }\end{array}$ & & $\begin{array}{c}\text { Iniciador } \\
\text { P-PO33-1 }\end{array}$ & $\begin{array}{c}\text { Iniciador } \\
\text { P-PO33-2 }\end{array}$ \\
\hline Patrones electroforéticos & 5 & 9 & & 7 & 5 \\
Índice diversidad & 0,64 & 0,83 & & 0,65 & 0,504 \\
$\mathrm{~N}^{\circ}$ total de alelos & 5 & 9 & & 8 & 8 \\
Promedio de bandas/genotipo & 3,3 & 5,7 & & 3,2 & 2,8 \\
\hline
\end{tabular}

\section{Conclusión}

En el estudio realizado en los genotipos selectos se encontraron diferencias significativas en la actividad de PPO y el IO, lo que permitió distinguir individuos con mejor comportamiento frente al PE. Asimismo, se observó variabilidad genética en las regiones de los genes de PPO amplificados. Sin embargo, hasta el momento no se pudo establecer asociación entre bandas y la actividad de PPO o IO. En el futuro, se prevé plantar material libre de virus en ambientes propicios para el cultivo de papas nativas y obtener mayor número de tubérculos y de mejor calidad para disminuir la incidencia del estrés biótico y abiótico y obtener así resultados contundentes.

\section{Agradecimientos}

Este trabajo está financiado para su realización por los proyectos PIP'08 No 6042 (CONICET), PICT'05 No 34878 (SECyT) y AETA No 282811 (INTA). Por otro lado, agradecer al Ing. Agr. Pablo Cicore por el análisis estadístico realizado, al Dr. Daniel Caldiz y a la Ing. Agr. Carolina de Lasa (MsC) por el aporte de la Carta de Color y a los Licenciados Leandro Barreiro, Martín Carboni y Juan M. D'ambrosio por la colaboración técnica.

\section{Literatura citada}

Álvarez-Mayorca, M. 2001. Oportunidades para el Desarrollo de Productos de Papas Nativas en el Perú. Revista Latinoamericana de la Papa. Vol. especial 2001: 58-79.

Clausen, A.M. 1989. Collecting indigenous potato varieties in Northwest Argentina. FAO/IBPGR Plant Genetic Resources Newsletter 80:38-39.

Computational Biology and Functional Genomics Laboratory. 2008. Solanum tuberosum Gene Index .En: Dana -Farber Cancer Institute and Harvard School of Public Health (http: //www.compbio.dfci.harvard.edu/tgi/plant.html) Versión 12.0, Julio 24 del 2008. Consulta Enero, 2010.

Chen, B., Zhou, G.Y., Wang, R.H. 2000. Study on heredity of enzymes for polyphenol-oxidase in rice and resistance to bacterial blight. Journal of South Agricultural University. 21(2): 46-48.

Friedman, M. 1997. Chemistry, biochemistry and dietary role of potato polyphenols. A review. Journal of Agricultural and Food Chemistry 45 (5): 1523-1540.

Hunt, M., Eannetta, N., Yu, H., Newman, S., Steffens, J. 1993. cDNA cloning and expression of potato polyphenol oxidase. Plant Molecular Biology 21: 59-68. 
Infostat, 2008. Infostat versión 2008. Manual del Usuario. Grupo Infostat, FCA, Universidad Nacional de Córdoba. Primera Edición. Editorial Brujas, Argentina.

Milbourne, D., Meyer R., Bradshaw, J.E., Baird, E., Bonar, N., Provan, J., Powell, W., Waugh, R. 1997. Comparison of PCR based marker systems for the analysis of genetic relationships in cultivated potato. Molecular Breeding 3: 127136.

MDE $^{\circledR}$ Gel Solutions. 2003. Protocols for SSCP and Heteroduplex Analyses. Cambrex Bio Science Rockland, Inc. En: http://www.cambrex.com/bioproductos.htm, 8 p. Consulta Marzo 2010.

Pätzold, Ch. 1973. Storage conditions and quality of potato tubers. ISHS Acta Horticulturae 38, Symposium on Vegetable Storage. The International Society for Horticultural Science. Porcel, L.; Routaboul, J.; Cheynier, V.; Lepiniec, L. and Debeaujon, I. 2006. Flavonoid oxidation in plants: from biochemical properties to physiological functions. Plant Science 12(1):2936.
Thygesen, P., Dry, I., Robinson, S. 1995. Polyphenol oxidase in potato. A multigene family that exhibits differential expression patterns. Plant Physiology 109:525-531.

Werij, J., Kloosterman, B., Celis-Gamboa, C., Ric de Vos, C., America, T., Visser, R., Bachem, Ch. 2007. Unravelling enzymatic discoloration in potato through a combined approach of candidate genes, QTL, and expression analysis. Theoretical and Applied Genetic 115:245-252. 